\title{
A Model Reduction Approach for Simulation of Long-term Voltage and Frequency Dynamics
}

\author{
Tilman Weckesser \\ Dept. Elec. Engineering \& Comp. Sc. \\ University of Liège, Belgium \\ j.weckesser@ulg.ac.be
}

\author{
Valeri Franz Eckhard Grebe \\ Amprion GmbH, Germany \\ valeri.franz@amprion.net \\ eckhard.grebe@amprion.net
}

\author{
Thierry Van Cutsem \\ Fund for Scientific Research (FNRS) \\ University of Liège, Belgium \\ t.vancutsem@ulg.ac.be
}

\begin{abstract}
This paper presents a novel approach, which identifies a buffer zone around a given study system and replaces the external system with an equivalent. The aim is to obtain a reduced system, which is suitable for assessment of long-term voltage and frequency dynamics in the study system. First, the proposed methodology ensures that all components, which are impacting the voltage profile of the study system, are included in the buffer zone. For that purpose, the propagation of reactive power changes from the boundary of the study system into the external system are used in a modified depth-first search algorithm to identify the buffer zone. Second, the external system is replaced with equivalents accounting for inertia and primary frequency control effects. The proposed method was successfully tested on a modified version of the ENTSO-E Dynamic Study Model.
\end{abstract}

Index Terms-model reduction, equivalent, dynamic security assessment, long-term voltage dynamics, frequency dynamics

\section{INTRODUCTION}

Globalization of the electricity market is increasing the share of power that is traded across national and regional borders. In order to remove trading bottlenecks, individual (e.g. national) power systems have stronger interactions with their neighboring systems. When assessing stability of an individual power system, it and its neighbors need to be represented with suitable dynamic models. However, carrying out dynamic security analysis on the full system model is impractical. Stability and security studies are usually carried out with focus on a certain part of the power system, which, in the following, is called study system. Generally it can be of interest to retain a buffer zone around the study system. The rest of the power system is commonly referred to as external system. The size of the dynamic model, to be considered in the analysis, can be reduced by replacing the external system with equivalents. It is crucial that these processes retain the effect of the external system on the study system.

Various static and dynamic network equivalents have been proposed in the past and can roughly be divided into two groups (see e.g. [1]). One group of methods solely utilizes measurements from the study system and its boundary. These require no knowledge about the configuration as well as parameters of the external system (for example [2], [3]).

Research supported by Amprion GmbH, Germany. Klaus Vennemann and Dr. Roland Becker are gratefully thanked for their assistance and their expert insights.
The approaches in the second group are often referred to as model reduction methods. They generally require knowledge about the external system and the resulting equivalents can be suitable for either static or dynamic studies.

One example for static studies is the so-called Ward equivalent, which was introduced in [4] and further developed in [5]. The elimination of all external buses is enabled by converting the generators and loads of the external system into constant current injectors. An extension of the equivalent, which allows steady-state assessment of generator outages, is introduced in [6]. For that purpose, the effect of the speed governors of external generators was included in the derivation of the Ward equivalent. The generalized Ward equivalent, which allows to mimic changes in reactive power injection from the external system, was presented in [7]. The authors of [8] proposed a dynamic Ward equivalent for transient stability analysis. It allows to eliminate all load buses in the external system, where voltage dependent loads are connected.

Another example is the REI (Radial, Equivalent and Independent) method, which was first discussed in [9]. It aims at replacing a set of nodes by one new equivalent node. In order to perform the network reduction, a zero loss network is setup, which connects all generator and load buses of the external system with a new fictitious bus. After the zero loss network is set up, the generators and loads of the external system are merged at a new fictitious bus and the external buses are eliminated. In [10] a dynamic REI equivalent suitable for short circuit analysis and transient stability assessment was presented. In this case REI equivalents are connected directly at the boundary buses of the study system. Then equivalent generators of coherent generator groups are defined and their model parameters are determined through participation factors. Several other approaches were developed for aggregation of coherent generators. An approach based on least-square fitting of the transfer functions of coherent generators was proposed in [11]. A trajectory sensitivity method was used in [12] to determine the exciter parameters of the aggregated generators. A structure preserving technique to compute parameters of an equivalent generator was discussed in [13].

In this paper, a new, practical method is proposed to reduce an original, large model of a power system while preserving the accuracy of time-domain simulations of long-term voltage and frequency dynamics. 


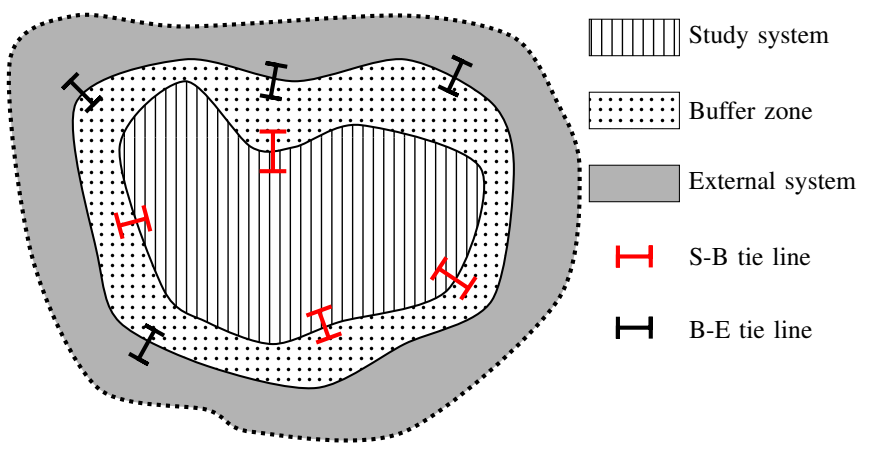

Fig. 1. Split of network into study system, buffer zone and external system.

For that purpose, a buffer zone around the given study system is determined as illustrated in Fig. 1. The topology and the components of the original system are preserved in the buffer zone (and study system). This ensures that the impact of the outside system on voltages in the study system is retained. Next, the external system enclosing the buffer zone is replaced with a suitable equivalent.

The rest of the paper is structured as follows. In Section II the method for identifying the buffer zone is presented and the dynamic equivalent used to replace the external system is described. Following, simulation results are reported in Section III on a modified version of the ENTSO-E Dynamic Study Model (DSM) [14]. Finally, concluding remarks are offered in Section IV.

\section{METHOD}

\section{A. Buffer zone identification}

Due to the strong relation between reactive power and voltage, the proposed method assesses the propagation of reactive power changes at the study system's boundary buses into the outside system, in order to identify the buffer zone. The boundary buses of the study system are those which have direct connections to buses in the outside system.

Due to the investigation of the propagation of reactive power changes, the size of the buffer zone is also dependent on how close the generators outside of the study system are to their respective reactive power limits.

The approach is explained with the help of pseudocode in Fig. 2; the text refers to particular line numbers in the algorithm $(A L)$.

The function IDENTIFYBUFFER receives as input network data, such as bus and branch data, and a list of the buses in the study system. The output of the function is the list of buses in the study system and the buffer zone, which is stored in explored.

In the first step, the function EXTRACTB is called, which identifies the boundary buses of the study system ( $A L 2)$. The inputs to the function are network topology data, such as bus, transmission line and transformer data, as well as the list of buses in the study system. EXTRACTB then returns a list of boundary buses. Afterwards, individually at each boundary bus a change of reactive power $\Delta Q_{\text {dis }}$ is simulated with the help of a simple power flow computation $(A L 4)$.

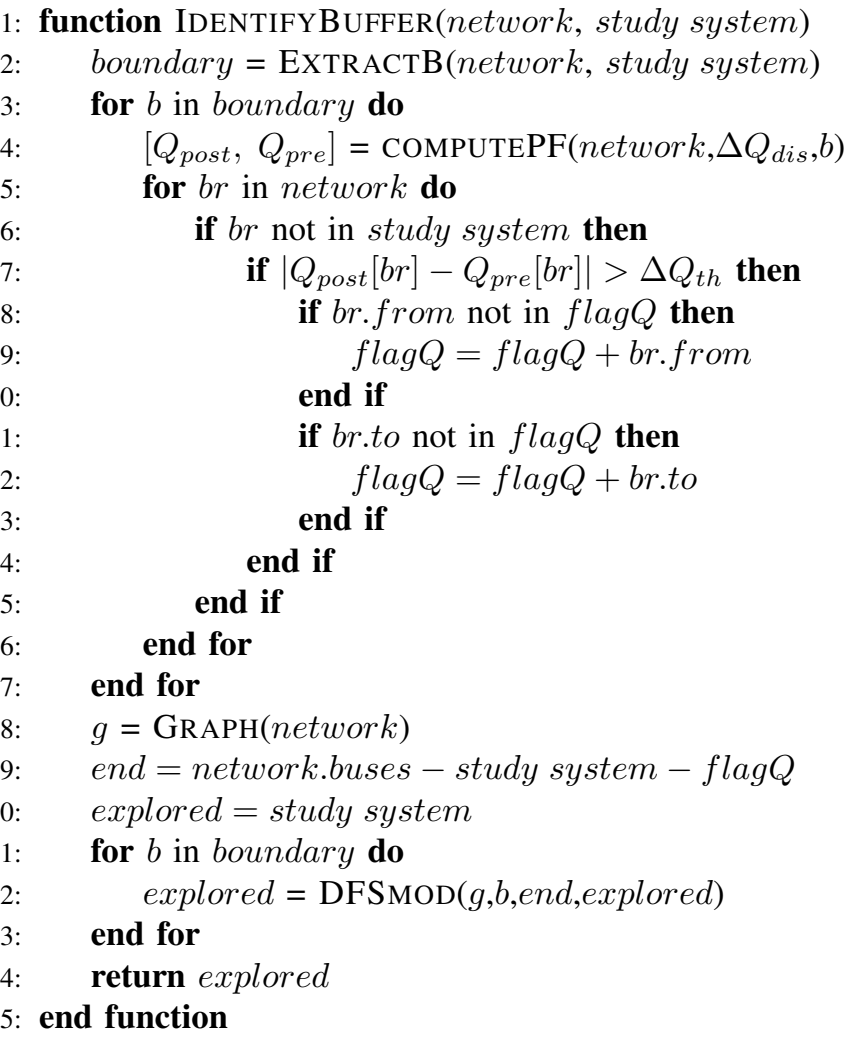

Fig. 2. Pseudocode of buffer zone identification.

Its propagation into the branches outside the study system is investigated $(A L 5-16)$. If the flow changes by more than a threshold $\Delta Q_{t h}$ (e.g. $5.0 \%$ of $\Delta Q_{d i s}$ ), the buses at both ends of the branch are marked as affected (AL9 and $A L 12$ ). Figure $3 \mathrm{a}$ illustrates the procedure on a fictitious system. Part of the non-study system is shown, where the buses are depicted with circles. The colors of the buses and lines indicate if they have been identified as being affected or not.

Subsequently, the power system is converted into a graph $g$ (AL18) utilizing the network data, where the nodes of the graph represent buses and the edges network branches. The aim is to include all affected buses, which are directly or through other affected buses connected to the study system. Affected buses only surrounded by non-affected buses are not added to the buffer zone (see Fig. 3b). This can be achieved with a slightly modified version of a recursive depth-first search (DFS) algorithm (see e.g. [15]). The DFSMOD (AL22) function receives a graph $g$, a start bus $b$, a list of end buses and optionally a list of explored buses. Beginning at the start bus the function explores the graph and terminates when all paths originating from start reached an end bus. The function then returns a list of all explored buses, which also includes the found end buses. (In contrast, the original DFS is terminated immediately, when the first path to an end bus is found.)

The list of end buses is initialized to contain all buses of the power system which are neither in the study system 


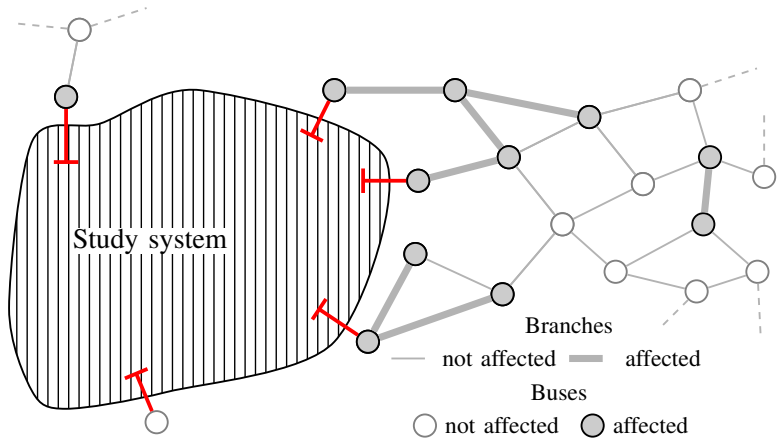

(a) Starting point of the modified DFS algorithm.

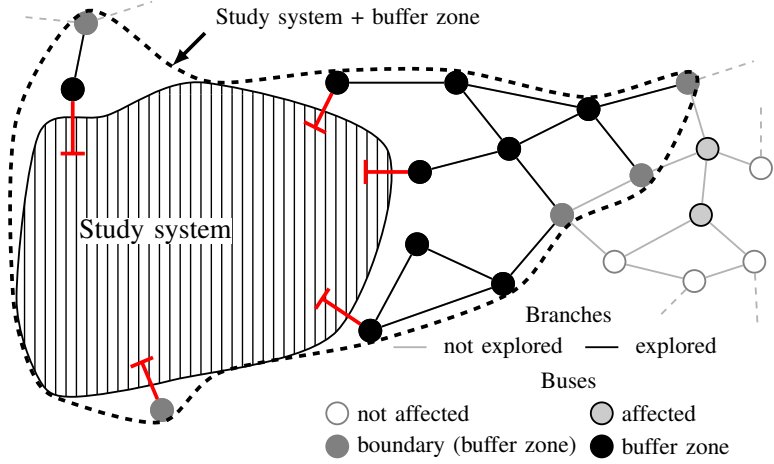

(b) Buffer zone identified with proposed algorithm.

Fig. 3. Visualization of buffer zone identification.

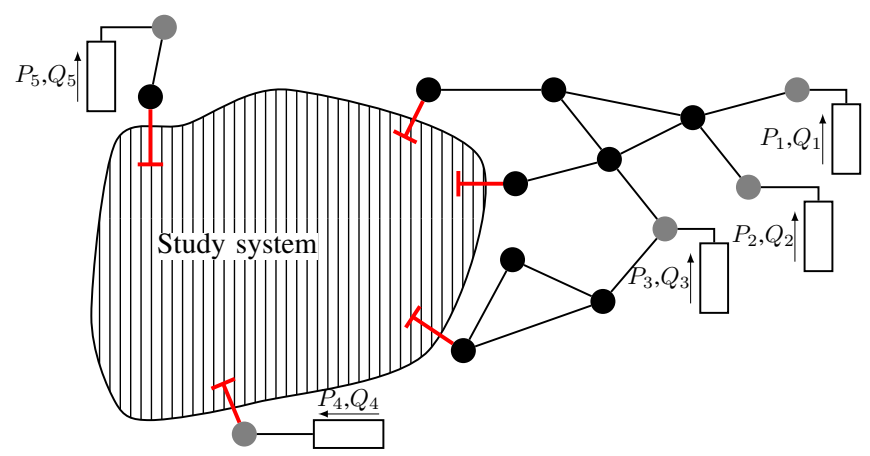

Fig. 4. DPIs are placed at each boundary bus.

nor flagged as affected (AL19). Since all the buses in the study system should be retained, the list of explored buses is initialized with it $(A L 20)$. Following, the function iterates through all boundary buses of the study system and executes the modified DFS providing the respective boundary bus $b$ as start bus $(A L 21-23)$. As a result, the final list of explored buses contains the buses of the study system plus all buses that should be part of the buffer zone.

Figure $3 \mathrm{~b}$ shows the result of the buffer identification algorithm. All explored buses (filled black and filled dark gray) around the study system form the buffer zone. Moreover, two affected buses (on the far right), which do not fulfill the requirement of being, directly or indirectly (through other affected buses) connected to the study system are not part of the buffer zone.

\section{B. Equivalencing the external system}

After the buffer zone has been identified, the external system is replaced by an equivalent aimed at reproducing its contribution to frequency dynamics. To this purpose, Dynamic Power Injectors (DPIs) as described in [16] are used to replace the external system and emulate its behavior in long-term dynamic simulations. DPIs are connected to all boundary buses of the buffer zone. Figure 4 shows the previous example, where now the external system has been replaced by DPIs. The procedure detailed in Section II-A ensures that all components of the nonstudy system, which are contributing to reactive power changes

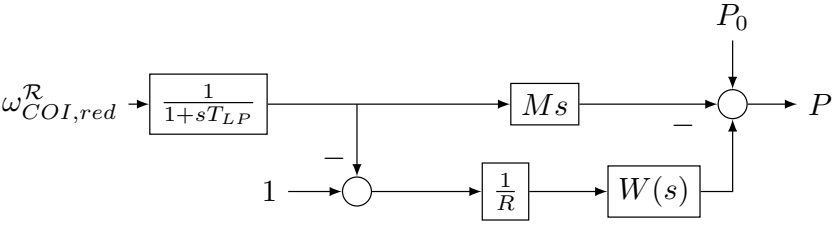

Fig. 5. Block diagram of the DPI in pu.

in the study system are included in the buffer zone. Since the boundary buses are the first buses where the reactive power change was negligible, the reactive power injected by each DPI remains constant, equal to its initial value. On the contrary, to reproduce the inertial and primary frequency control effects of the external system, DPIs modulate their active power injection after a disturbance. To this purpose, each DPI uses as input the Center Of Inertia (COI) speed of the reduced system:

$$
\omega_{\text {COI,red }}^{\mathcal{R}}(t)=\frac{\sum_{i \in \mathcal{R}} \omega_{i}(t) \cdot M_{i}}{\sum_{i \in \mathcal{R}} M_{i}}
$$

where $\omega_{i}$ is the rotor speed of the $i$-th machine, $M_{i}$ the corresponding inertia constant, and $\mathcal{R}$ the set of all machines in the study system and buffer zone.

Figure 5 shows the block-diagram model of one DPI. The initial active power $P_{0}$ is obtained from the sum of power flows in the branches connecting the boundary bus of concern to other buses in the buffer zone or study system. The active power is modulated in response to changes of $\omega_{C O I, \text { red }}^{\mathcal{R}}$. A simple low-pass filter is used to cut off the undesired higher frequency oscillations in the signal. The parameter $M$ corresponds to an equivalent inertia constant and $R$ to the speed droop. The transfer function $W(s)$ defines the dynamics of the primary frequency control of the DPI. In this paper, the following simple transfer function is assumed:

$$
W(s)=\frac{1+s T_{z}}{1+s T_{p}}
$$

In order to retain the dynamic response of the unreduced system, the parameters of the equivalents are tuned. A simple approach to determine one set of parameters suitable for all connected DPIs was presented in [16]. 
TABLE I

SCENARIO $I I$ - SEQUENCE OF EVENTS

\begin{tabular}{l|c|l} 
Time & Name & Description \\
\hline \hline $1.0 \mathrm{~s}$ & $C 1$ & Tripping of first transmission line \\
\hline $1.5 \mathrm{~s}$ & $C 2$ & Tripping of second transmission line \\
\hline $2.0 \mathrm{~s}$ & $C 3$ & Tripping of first generator \\
\hline $2.5 \mathrm{~s}$ & $C 4$ & Tripping of second generator
\end{tabular}

TABLE II

COMPARISON OF THE SIZE OF THE BUFFER ZONE DEPENDING ON THE CHOSEN REACTIVE POWER THRESHOLD $\Delta Q_{t h}$

\begin{tabular}{c|c|c|c|c|c}
$\Delta Q_{t h}$ in $\%$ & 1.0 & 2.5 & 5.0 & 10.0 & 25.0 \\
\hline \hline study + buffer & 8067 & 7459 & 7059 & 6850 & 6563 \\
\hline Buffer zone & 1677 & 1069 & 669 & 460 & 173 \\
\hline Boundary buses & 418 & 314 & 227 & 182 & 91
\end{tabular}

\section{Simulation RESULTS}

\section{A. Unreduced test system and scenario}

The test system is a modified version of the ENTSO-E Dynamic Study Model (DSM), which was originally presented in [14]. The study system was chosen to be Germany, some parts of which were represented in greater details for longterm dynamics studies. The model includes detailed generator excitation systems with overexcitation limiters, and Load Tap Changers (LTCs) with inverse-time characteristics on transformers feeding loads. All loads are represented as $100 \%$ constant current and $100 \%$ constant admittance for the active and reactive power components, respectively. The model comprises ca. 27000 buses, 35000 branches and 900 generators.

In the following, results from two scenarios are presented. Scenario $I$ involves an $N-1$ contingency, namely the tripping of a generator producing around $1200 \mathrm{MW}$ in the study system. Scenario II was selected to assess the accuracy of the equivalent, when analyzing an $N-k$ contingency. The simulation involves an $N-4$ contingency consisting of tripping two generators and two transmission lines in the study system, taking place sequentially as shown in Table I.

\section{B. Buffer zone identification}

The study system consists of ca. 6400 buses and has 42 boundary buses. In order to investigate the dependency of the buffer zone on the chosen reactive power threshold, the algorithm (shown in Fig. 2) was tested with $\Delta Q_{t h}$ varying between 1 and $25 \%$ of the applied reactive power disturbance $\Delta Q_{d i s}$ chosen to be 200.0 Mvar. Table II shows the size of the study system including the respective buffer zone, the size of the buffer zone itself and the number of its buses as a function of $\Delta Q_{t h}$. The size of the buffer zone varies between 173 buses $\left(\Delta Q_{t h}=25 \%\right)$ and 1677 buses $\left(\Delta Q_{t h}=1 \%\right)$. As expected, this size and the number of boundary buses increases, when the threshold is decreased.

In the remaining of the paper, results are compared using the buffer zone determined with $\Delta Q_{t h}=5 \%$ and $\Delta Q_{t h}=1 \%$. These two variants are referred to as Case 1 and Case 2, respectively.
TABLE III

TUNED DPI PARAMETERS IN BOTH CASES.

\begin{tabular}{c|c||c|c|c|c|c} 
Case & \#DPIs & $T_{L P}[\mathrm{~s}]$ & $M[\mathrm{~s}]$ & $R$ & $T_{z}[\mathrm{~s}]$ & $T_{p}[\mathrm{~s}]$ \\
\hline 1 & 227 & 0.622 & 186.8 & 0.024 & 2.5 & 9.8 \\
\hline 2 & 418 & 0.622 & 87.1 & 0.048 & 2.5 & 9.8
\end{tabular}

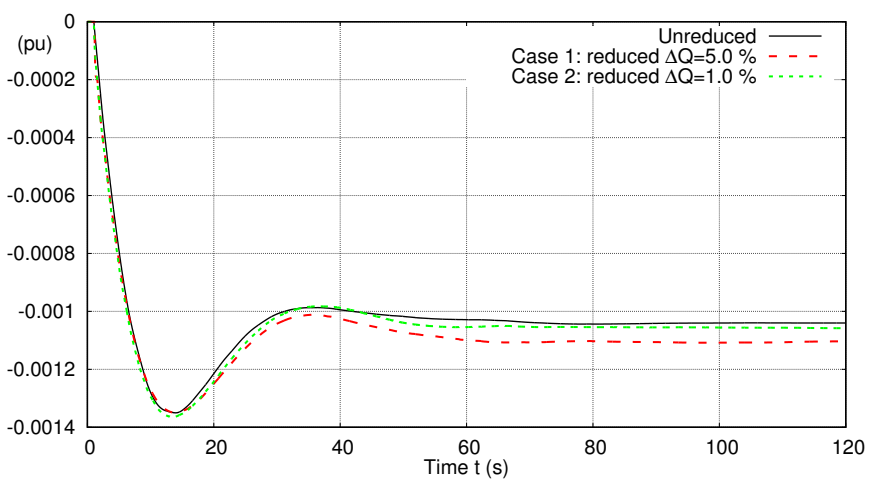

Fig. 6. Scenario $I$ - Speed deviation of COI in the unreduced system and reduced system.

\section{Equivalent DPIs}

The parameters $R, M, T_{z}$ and $T_{p}$ were determined with the approach presented in [16] and the resulting values are shown in Table III. In Case 2 the number of DPIs is almost twice as large as in Case 1, which is due to the increased size of the buffer zone and the resulting larger number boundary buses. Since in Case 2 the contribution of the external system to the inertial response as well as primary frequency response is now shared between a larger number of DPIs, the droop $R$ is doubled and the inertia constant $M$ is almost halved.

\section{Results of Scenario I}

As stated in Section II-B, the purpose of the equivalent is to reproduce the inertial and primary frequency control effects of the external system. Figure 6 shows a comparison of the speed deviation of the COI in the unreduced and the reduced system, respectively. It should be noted that, while in the unreduced system all machines are considered in the computation of the COI, in the reduced system only the synchronous generators in the study system and buffer zone are taken into account. The DPIs are not involved in the COI computation (but, of course, contribute to frequency support through the modulation of their active power). It can be observed that after $20 \mathrm{~s}$, the COI speed deviation in Case 1 slightly differs from that of the unreduced system, while it remains very close in Case 2. The final discrepancy is explained by small voltage deviations (inside the LTC deadband) that cause the load active powers to be slightly different in the reduced model.

Let us recall that the proposed approach also aims at providing a reduced model suitable for assessing long-term voltage dynamics in the study system.

Figure 7 compares the evolution of a bus voltage magnitude in the unreduced and reduced systems. The chosen bus is the 


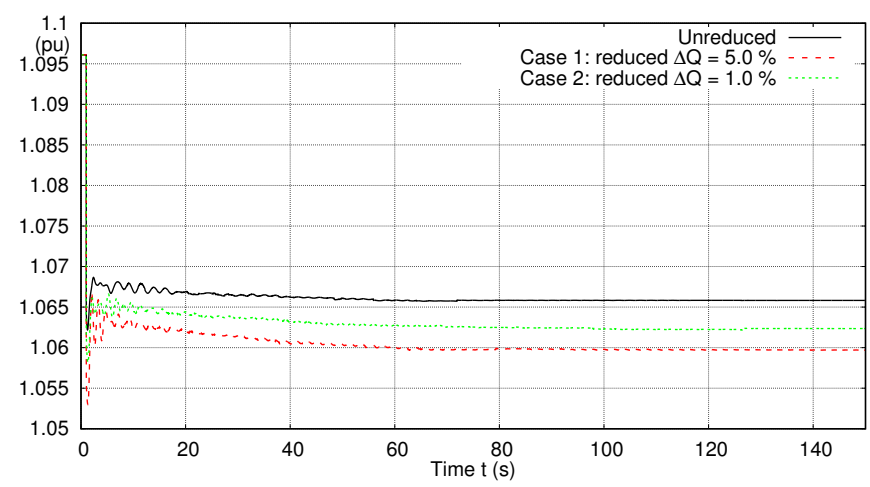

Fig. 7. Scenario $I$ - Comparison of voltage evolutions on the high-voltage side of the step-up transformer of the tripped generator.

high-voltage side of the step-up transformer of the tripped generator. In the simulation of the unreduced system, it can be observed that after the loss of the generator the voltage drops transiently to $1.062 \mathrm{pu}$ and eventually settles at $1.066 \mathrm{pu}$, which is approximately $0.03 \mathrm{pu}$ below the initial value. In Case 1 , the voltage drops down to $1.053 \mathrm{pu}$ and settles at a value of $1.060 \mathrm{pu}$, while in Case 2 it drops to $1.058 \mathrm{pu}$ and settles at $1.062 \mathrm{pu}$. Hence, the simulation with the equivalent is in both cases slightly more pessimistic, with a final voltage magnitude approximately $0.006 \mathrm{pu}$ and respectively $0.004 \mathrm{pu}$ lower. The results of Case 2 are closer to the reference than those of Case 1 as expected.

In order to evaluate the overall accuracy of the voltage evolutions in the reduced system, at each time step of the dynamic simulation and for each bus in the study system the following difference was computed:

$$
\Delta V_{i}(t)=V_{i}(t)-V_{r e d, i}(t)
$$

where $V_{i}(t)$ is the voltage magnitude at the $i$-th bus at time $t$ extracted from simulation of the unreduced system and $V_{\text {red,i }}(t)$ from the reduced system. Drawing up the minimum and maximum deviations defines an envelop, which encloses the $\Delta V_{i}(t)$ of all buses. It should be noted that this is a very strict test, since the $L_{\infty}$-norm of the error in each time step is considered. The corresponding graph is shown in Fig. 8 relative to all transmission buses in the study system. During the initial transients, the voltage magnitudes deviate between $-0.015 \mathrm{pu}$ and $0.011 \mathrm{pu}$ in Case 1 . In Case 2 a maximum difference of $\pm 0.006 \mathrm{pu}$ is observed. After the oscillations have damped out, the voltage magnitudes in the reduced and unreduced simulation deviate by no more than $0.009 \mathrm{pu}$ in Case 1, while in Case 2 the differences are in the range of $-0.003 \mathrm{pu}$ to $0.005 \mathrm{pu}$. This shows that by increasing the buffer zone the envelop width could be more than halved: from $0.018 \mathrm{pu}$ (Case 1) to $0.008 \mathrm{pu}$ (Case 2).

\section{E. Results of Scenario II}

Figure 9 shows a comparison of the COI speed deviations in the unreduced and the reduced systems. In the unreduced



Fig. 8. Scenario $I$ - Comparison of envelops of voltage magnitude deviation; unreduced vs. reduced systems.

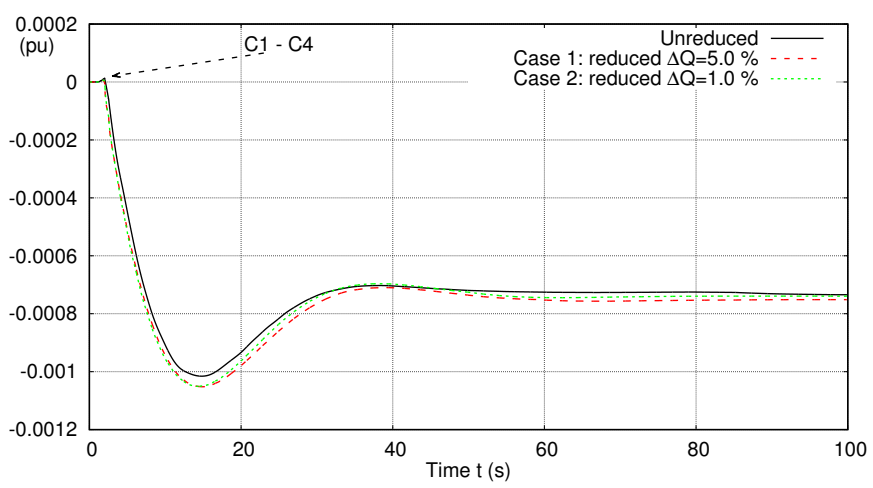

Fig. 9. Scenario $I I$ - Speed deviation of COI in the unreduced and reduced system.

system, the COI speed deviation drops after the sequence of contingencies to a minimum of $-1.0 \cdot 10^{-3} \mathrm{pu}$, before it settles at a new steady-state value of $-0.73 \cdot 10^{-3} \mathrm{pu}$.

When comparing the results of the unreduced and reduced system, a good agreement between the COI response of the unreduced and both reduced systems can be observed. In both reduced systems, the COI speed deviation drops to $-1.05 \cdot 10^{-3}$ pu slightly below that of the unreduced system. Eventually, the speed deviation settles at a $-0.75 \cdot 10^{-3} \mathrm{pu}$ in Case 1 and $-0.74 \cdot 10^{-3} \mathrm{pu}$ in Case 2 .

An example of long-term voltage evolution is provided in Fig. 10 relative to a transmission bus in the study system. After the short-term transients, the voltage evolves under the effect of LTC actions. Again the results of Case 2 are closer to the reference than those of Case 1 . They approach the reference even better than in Scenario $I$.

Similarly to Fig. 7, the bus voltage magnitude experiencing the largest drop was investigated. The drop was found to be: $0.08 \mathrm{pu}$ for the unreduced system, $0.085 \mathrm{pu}$ in Case 1 and $0.081 \mathrm{pu}$ in Case 2. The final voltage of Case 2 remains very close to that of the unreduced system.

Figure 11 shows the envelop for Cases 1 and 2, which involves the $\Delta V_{i}(t)$ of all transmission buses in the study system. The graph shows that right after the contingency 


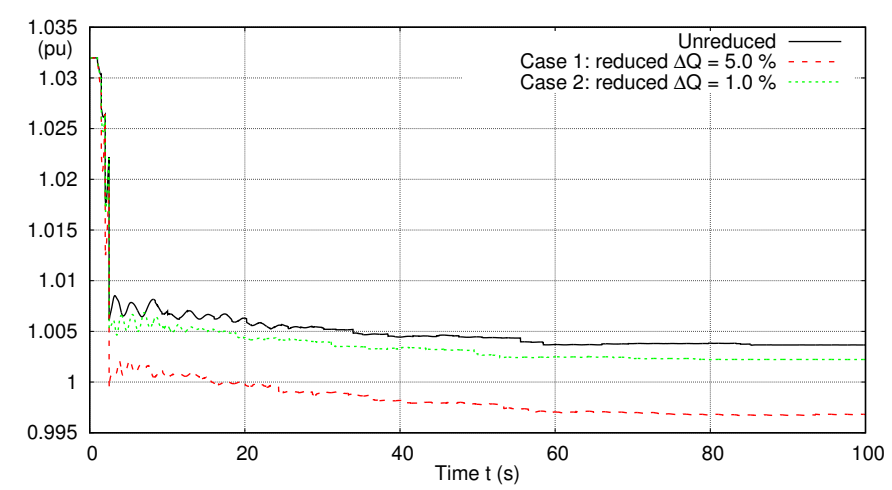

Fig. 10. Scenario $I I$ - Comparison of voltage evolutions at a transmission bus in the study system.

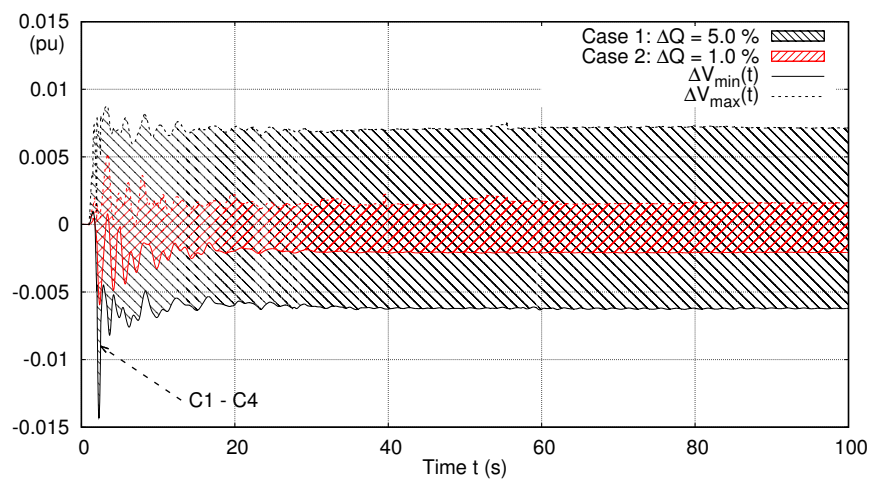

Fig. 11. Scenario II - Comparison of envelops of voltage magnitude deviations; unreduced vs. reduced systems.

occurrence, larger deviations take place. In Case 1, the deviations reach a maximum of $-0.014 \mathrm{pu}$. However, in the new steady state, the voltage deviations are in the range of $-0.006 \mathrm{pu}$ and $0.008 \mathrm{pu}$. In Case 2, the voltage deviations are considerably smaller. During the initial dynamics, the deviations reach maxima of $-0.006 \mathrm{pu}$ and $0.005 \mathrm{pu}$. After the transients have died out, the deviations decrease and lie in the range of \pm 0.002 .

\section{CONCLUSION}

In this paper a novel, practical method has been proposed to reduce the original, large model of a power system while preserving the accuracy of time-domain simulations of longterm voltage and frequency dynamics.

The approach considers a buffer zone around the study system in which the topology and the components of the original system are preserved. This buffer zone is identified by considering the propagation of reactive power changes from the study system boundary into the outside system.

Outside the buffer zone, the external system is simply replaced by dynamic power injectors connected to the boundary buses of that zone. Their reactive power injections are constant since the buffer zone and its components already provide the reactive power support. Whereas, their active power injections react to the COI speed of the machines in the study system and buffer zone. This emulates the contribution of the external system to inertial and primary frequency control effects.

The method has been tested on a modified version of the ENTSO-E dynamic study model, where an $N-1$ contingency and an $N-k$ contingency sequence were assumed. The timedomain simulation results show that the COI speed response of the reduced model closely matches the one of the unreduced system. They also show that, after the short-term dynamics have died out, the voltage magnitudes at transmission buses of the study system closely match the corresponding values obtained with the unreduced model. Finally, the results demonstrated that, the equivalent is suitable for the analysis of $N-k$ contingencies.

It must be emphasized that the proposed equivalent (in particular the dynamic power injector technique) does not preserve the original inter-area electromechanical oscillations. This could be the subject of further developments.

\section{REFERENCES}

[1] J. Machowski, J. W. Bialek, and J. R. Bumby, Power System Dynamics: Stability and Control, 2nd ed. John Wiley \& Sons, 2009.

[2] J. Dopazo, M. Dwarakanath, J. Li, and A. Sasson, "An external system equivalent model using real-time measurements for system security evaluation," IEEE Transactions on Power Apparatus and Systems, vol. 96, no. 2, pp. 431-446, 1977.

[3] X. Feng, Z. Lubosny, and J. W. Bialek, "Identification based dynamic equivalencing," in Power Tech, 2007 IEEE Lausanne, July 2007, pp. 267-272.

[4] J. B. Ward, "Equivalent Circuits for Power-Flow Studies," AIEE Transactions, vol. 68 , no. 1 , pp. 373-382, 1949

[5] A. Monticelli, S. Deckmann, A. Garcia, and B. Stott, "Real-time external equivalents for static security analysis," IEEE Transactions on Power Apparatus and Systems, vol. PAS-98, no. 2, pp. 498-508, 1979.

[6] F. Aschmoneit and J. Verstege, "An External System Equivalent for online Steady-State Generator Outage Simulation," IEEE Transactions on Power Apparatus and Systems, vol. PAS-98, no. 3, pp. 770-779, 1979.

[7] R. van Amerongen and H. van Meeteren, "A Generalised Ward Equivalent for Security Analysis," IEEE Transactions on Power Apparatus and Systems, vol. PAS-101, no. 6, pp. 1519-1526, jun 1982.

[8] T. Baldwin, L. Mili, and A. Phadke, "Dynamic Ward equivalents for transient stability analysis," IEEE Transactions on Power Systems, vol. 9, no. 1, pp. 59-67, feb 1994.

[9] P. Dimo, Nodal Analysis of Power Systems. Kent, England: Abacus Press, 1975

[10] F. Milano and K. Srivastava, "Dynamic REI equivalents for short circuit and transient stability analyses," Electric Power Systems Research, vol. 79, no. 6, pp. 878-887, 2009.

[11] A. J. Germond and R. Podmore, "Dynamic Aggregation of Generating Unit Models," IEEE Transactions on Power Apparatus and Systems, vol. PAS-97, no. 4, pp. 1060-1069, 1978.

[12] R. J. Galarza, J. H. Chow, W. W. Price, a. W. Hargrave, and P. M. Hirsch, "Aggregation of exciter models for constructing power system dynamic Equivalents," IEEE Transactions on Power Systems, vol. 13, no. 3, pp. 782-788, 1998 .

[13] M. Ourari, L.-a. Dessaint, and V.-Q. Do, "Dynamic Equivalent Modeling of Large Power Systems Using Structure Preservation Technique," IEEE Transactions on Power Systems, vol. 21, no. 3, pp. 1284-1295, 2006.

[14] A. Semerow, S. Hohn, M. Luther, W. Sattinger, H. Abildgaard, A. D. Garcia, and G. Giannuzzi, "Dynamic Study Model for the interconnected power system of Continental Europe in different simulation tools," in 2015 IEEE Eindhoven PowerTech. IEEE, jun 2015, pp. 1-6.

[15] J. Kleinberg and E. Tardos, Algorithm Design, M. Suarez-Rivas, Ed. Boston: Pearson Addison Wesley, 2006.

[16] T. Weckesser and T. Van Cutsem, "An Equivalent to Represent Inertial and Primary Frequency Control Effects of an External System," IET Generation, Transmission \& Distribution, in press. 